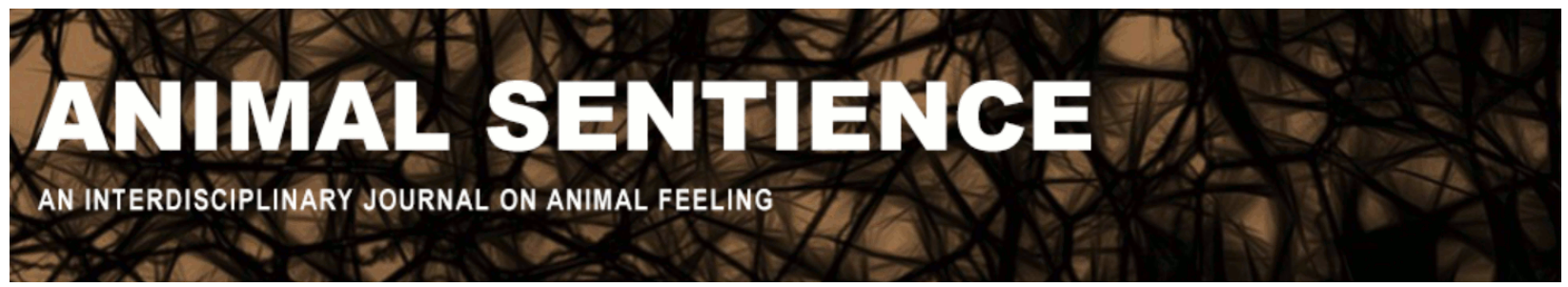

Cook, Peter F. and Berns, Gregory S (2018) The degeneracy of behavior and the rise of neuroimaging to measure affective states in dogs. Animal Sentience 22(23)

DOI: $10.51291 / 2377-7478.1372$

Date of submission: 2018-09-24

Date of acceptance: 2018-10-04

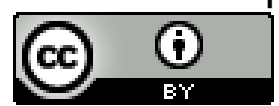

This article has appeared in the journal Animal

Sentience, a peer-reviewed journal on animal

cognition and feeling. It has been made open access,

free for all, by WellBeing International and deposited

in the WBI Studies Repository. For more information,

please contact

wbisr-info@wellbeingintl.org.

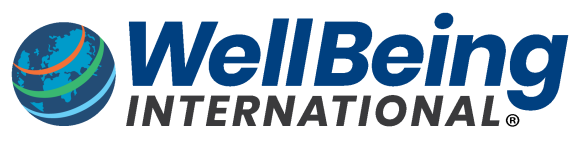

SOLUTIONS FOR PEOPLE, ANIMALS AND ENVIRONMENT 


\title{
The degeneracy of behavior and the rise of neuroimaging to measure affective states in dogs
}

Response to Commentary on Cook et al. on Dog Jealousy

\author{
Peter F. Cook ${ }^{1}$ \& Gregory S. Berns ${ }^{2}$ \\ ${ }^{1}$ Department of Psychology, New College of Florida, Sarasota, FL \\ 2Psychology Department, Emory University, Atlanta, GA
}

\begin{abstract}
It is gratifying and significant that so many scientists from diverse fields are arguing in-depth regarding a particularly complex set of social emotions in a non-human animal. Emotions play a fundamental role in decision making and information processing. Neuroimaging is important in understanding the cognitive and emotional worlds of non-human animals and can help measure covert emotions lacking clear behavioral correlates. Various experimental approaches could clarify the relative importance of attachment and aggression in jealousy and whether the phenomenon we measured is more akin to human envy or jealousy. Reverse inference from amygdala activation is probably justified because behavior is "degenerate": there are fewer behavioral programs than brain states that give rise to them. Individual differences are also important.
\end{abstract}

Peter Cook is Assistant Professor of Psychology at the New College of Florida. He studies animal cognition and comparative neuroscience in a wide range of species. He is particularly interested in finding novel, ecologically valid approaches to studying the brain and behavior outside the traditional laboratory setting. Website

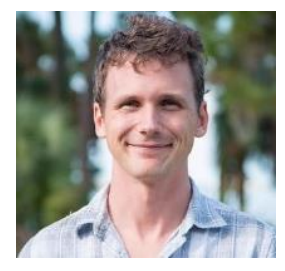

Gregory S. Berns, Distinguished Professor in Psychology, Emory University, directs the Facility for Education \& Research in Neuroscience. His research uses fMRI to study canine cognitive function in awake, unrestrained dogs to map their brains' perceptual and decision systems non-invasively. He also uses diffusion tensor imaging (DTI) to reconstruct the white matter pathways of a wide variety of other mammals, including dolphins, coyotes, and the extinct Tasmanian tiger. Website

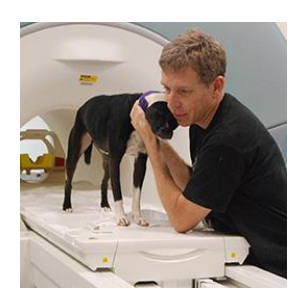

\section{Introduction}

In our target article (Cook et al., 2018) , we presented functional brain imaging evidence for dogs' sensitivity to social interactions between a familiar human and unfamiliar dog. Dogs were trained to lie still in an MRI machine and then watched their owner provide food to either a bucket or a realistic fake dog. We compared BOLD response in each dog's amygdala in these two conditions. Using previously gathered data on temperament, we determined that dogs who were more prone to aggression showed greater amygdala activation when their owner interacted with the fake dog than with the bucket. Our interpretation of these data was careful and conservative. In suggesting that the aggressive dogs' brain activations 
showed a response to social resource threat, we drew on prior behavioral findings that dogs respond more strongly when the owner interacts with a fake dog than with a neutral inanimate object. We also relied on well-established cross-species links between amygdala activation and physiological arousal. Because the fake dog was always present during the experiment, the apparent increased arousal in some dogs when their owners interacted with the fake dog seemed compelling evidence of socioemotional sensitivity. Although the title of our target article raised the specter of jealousy, we were careful to distinguish what we measured from full-blown human jealousy and favored the term "proto-jealousy." Nevertheless, we believe that our work builds on similar prior behavioral work on how dogs react to observed social interactions. The socioemotional world of the dog, our most common animal companion, is of great theoretical and practical interest.

The commentaries on our findings and interpretation have been widely varied and highlight the breadth and depth of current thinking on animal emotion and cognition, particularly as it relates to the domestic dog. We thank each of the commentators for their time and care, and for furthering this ongoing discussion. Before addressing specific points, we would like to emphasize that most of the commentaries engaged with our arguments at the level of interpretation. Many acknowledged the novelty of the results but were not always in agreement with our specific framing of our findings. For example, a number of commentators suggested that perhaps we were measuring something more akin to protoenvy than proto-jealousy. Others questioned whether our focus on aggression - as opposed to attachment - was appropriate for examining jealousy and related emotional phenomena. Others questioned whether the amygdala was the right locus for such an inquiry. In each of these cases, there are good-faith arguments to be made, and productive debate to be had. We stake some of our own ground on these matters below, although in many cases, a definitive answer will require more data. In a number of cases, commentators have suggested clever follow-ups that are likely to be productive and may help clarify some of these matters.

It is notable that so many engaged scientists are discussing how best to understand the socioemotional world of the dog, as opposed to arguing over whether dogs are likely to have any subjective or complex emotions in the first place. We hold this to be meaningful evidence of progress in comparative psychology, affective neuroscience, and canine research. While many weighty questions remain about subjective experience, and the extent to which it is, or is not, shared across species, there is increasing availability of the kinds of technological tools and experimental wherewithal to make meaningful measurements of neurobiological and peripheral changes that we strongly believe to be linked to emotion. Human emotions are rooted in activations in the peripheral and central nervous system. We agree with Webb and de Waal, that as we gain increasing understanding of the relevant mechanisms and develop more broadly applicable and finer gained tools for examining these phenomena, we can expand the scope of meaningful inquiry out into the animal kingdom. Our science and our relationship with the animal world will only benefit.

\section{Proto-Jealousy or Proto-Envy?}

Some commentators believed we were assessing something more akin to envy than to jealousy (Harmon-Jones \& Harmon-Jones; Vonk; Zentall). The claim is that envy is a negative emotion inspired by desiring material goods or traits possessed by another (e.g., Smith \& Kim, 2007), while jealousy is a negative emotion inspired by loss or threatened loss 
of social access to a conspecific (e.g., White \& Mullen, 1989). We do not claim that we measured jealousy in dogs, per se, but rather that we assessed neural evidence of covert arousal in response to social resource threat. This may be related to jealousy in humans, but we don't claim it is identical. That aside, the question remains whether the resource our dogs lost was food or social interaction, and how these might be differentially related to jealousy as opposed to envy. Our focus on the interpersonal component of the response, specifically referring to social resource threat, is because in both the fake dog and the bucket condition, the subject loses access to food. It is the social component of the fake-dog condition that would seem to differentiate it. However, we did not experimentally isolate loss of food to a conspecific due to the action/presence of the human versus a non-social agent. An interesting follow-up would assess differential response when the food is distributed by a human versus a mechanical apparatus (as suggested by Harmon-Jones \& Harmon-Jones). Covert arousal when it is either a fake dog or a bucket receiving the food in a non-social context suggests something more akin to what our commentators prefer to interpret as envy. Different responses when the fake dog receives food from the human versus a mechanical apparatus might suggest something more akin to what might be interpreted as jealousy. We have no quibble with this labeling. We merely note that both envy and jealousy are highly social emotions that are of interest from a comparative perspective.

\section{Is There Such a Thing as "Covert Arousal"?}

In our target article, we suggested that dogs with increased amygdala activation in the owner-feeds-fake-dog condition may have been in a state of covert arousal. Some might question whether "covert arousal" is a coherent concept. By relying on amygdala activation as an indicator of emotional state, we are attempting to measure emotion in dogs without an explicit behavioral correlate. Indeed, our subjects need to sit still during scanning; our entire project depends on the feasibility of such measures. As noted by van Kleef, emotions have long been identified with overt social signaling behavior - a smile, bared teeth, a falling tear. Some consider emotions to be fundamentally social constructs (Parkinson, 1996; van Kleef, 2009). Aggression would then be a signal to a conspecific that one is prepared to fight; in the absence of that signaling, the emotion must not be inferred.

In social species, many emotions are indeed socially relevant and hence frequently linked to social signals. However, we would strongly reject an argument that emotions are primarily and predominantly social or require external signaling. From the perspective of affective neuroscience, emotions evolved to inform and restrict choice in animals with flexible behavior (Lerner et al., 2015). That is, they have an information processing function. Central representations of peripheral body states are made available to cognitive and decision-making systems (Panksepp, 2004). Arguably, the processing of these representations is the stuff of emotion. As suggested by Ekman (1992), echoing William James, "If basic emotions evolved to deal with fundamental life-tasks, they should not only provide information through expressions to conspecifics about what is occurring, but there should also be physiological changes preparing the organism to respond differently in different emotional states." Social emotions, and the social signaling of emotions, are just part of a much larger construct. An aggressive impulse may increase the likelihood that an animal will act in an overtly aggressive manner. But an aggressive impulse, which constitutes 
some form of internal emotional state, may fail to reach a threshold for aggressive action, or may be actively inhibited in a top-down manner (e.g., Apter et al., 1990).

We certainly accept that a human might be jealous and choose not to act based on that emotion, for fear of embarrassment, or endangering a relationship. From our perspective, it is perfectly coherent to suggest that a dog may be experiencing arousal or aggressive impulses that it does not act on. The dogs in our studies are well-trained to remain still during scanning, and we have previously successfully located frontal regions contributing to the inhibition of prepotent motor response in these dogs (Cook, Spivak \& Berns, 2016). The question of particular interest is when, and in what situations, an internal agitation might manifest as overt behavior. We suggest that we are identifying dogs who are more likely to act (aggressively or otherwise) in situations involving a social resource threat. Presumably these dogs are more sensitive to such threats. As suggested by Howell, physiological measures might be used to help monitor socialization.

\section{Does Jealousy Rely on Attachment?}

A number of commenters question our reliance on the aggression scales in the C-BARQ temperament assessment, suggesting that attachment might be a better construct to examine in relation to jealousy or proto-jealousy (Harmon-Jones \& Harmon-Jones; Overall; Serpell). This may seem intuitive, in that dogs with more "secure" attachment to their owners might be less aroused by a potential social threat. However, we found no correlation with attachment or attention-seeking. Moreover, the human literature suggests that attachment style does not necessarily mediate the intensity of jealous emotions. It mediates the targets of those emotions (toward either the target of affection (secure) or the interloper (insecure) (Sharpstein \& Kirkpatrick, 1997). Given the importance of the primary human bond, it is plausible that even securely attached dogs would be sensitive to a social threat, although the target of their arousal and their resultant behavior might differ in interesting ways. Our paradigm did not allow us to differentiate components of the dogs' reaction to the human-fake-dog interaction, but future studies might do so. In addition, the human literature clearly links jealousy and aggression (Culotta \& Goldstein, 2008; Grotpeter \& Crick, 1996). Irrespective of whether attachment style has an effect on dog social resource guarding, it remains plausible that arousal, amygdala activation, and proneness to aggressive behavior are also relevant.

\section{Why Bother with Neuroimaging?}

We are now in the third decade since fMRI was first described, and there has been a veritable gold rush to neuroimaging as a noninvasive way to peer into the brain of (primarily) humans but sometimes other animals. And while there has been periodic backlash against overinterpretation of imaging results, fMRI is still the best tool available for measuring taskrelated brain activity as well the increasingly popular resting-state. In our view, there are two distinct reasons for using neuroimaging.

First, fMRI is used to elucidate the neural mechanisms associated with known behavioral or mental phenomena. In the simplest use, this might answer the question of where in the brain a process takes place. The literature is filled with such brain-mapping studies; this has been pejoratively termed "blobology" or "neural phrenology." However, 
identifying the potential loci of neural processes is the first step in understanding the link between brain activity and mental phenomena. Research in the visual system, for example, has been particularly productive in mapping the locations of face-sensitive regions, object areas, and other regions associated with spatial navigation. Once identified, further research and debate continues about how these regions work together and which aspects of visual perception they implement.

The second use of neuroimaging is more exploratory. Many mental phenomena are not as easily quantified as visual perception. Emotional states are particularly tricky because their behavioral manifestations may be highly variable. The Cartesian notion that only "jealousy behavior" can be studied (Abdai \& Miklósi; Nagel, 1974) relegates the dog's mind to the back seat and echoes others who have dodged the hard problem of subjective experience (LeDoux, 2014). In the human literature, self-report is the usual standard, although this suffers from reliability problems as well. Within this space, neuroimaging offers potentially more objective biomarkers of internal states. The growing use of machinelearning has hinted at this possibility. But before we get to the point of full-on braindecoding, we must tackle the thorny issue of reverse inference.

As we made clear in the target article, "Amygdala activation should not be equated with specific emotions." Herein lies the problem of reverse inference. As several commentators also noted (Adolphs; Harmon-Jones \& Harmon-Jones; Jiang et al.), it is not generally possible to infer a specific emotional state from the activity in a single brain region. It should be noted that the initial skepticism about reverse inference was due in part to the selection of Broca's area as the test case for language processing. As it turned out, that region is indeed involved in a wide variety of executive functions, so it should be no surprise that it wasn't completely predictive of language processing per se. However, other brain regions have turned out to have impressive reliability for predicting specific cognitive processes (Poldrack, 2006; Poldrack, 2011). The establishment of the neurosynth database (neurosynth.org) has made it straightforward to check. For example, the reverse-inference Z-score of ventral striatum activity with "Reward" is 25 ! This means that in the published literature, there is a very high likelihood that when ventral striatum activity is reported, some reward process is active. For comparison, Broca's area presently has a reverse Z-score of about 12 for language.

So what about the amygdala? Unfortunately, "jealousy" does not appear in the neurosynth database. Nor does "envy." But "anger" has a reverse inference Z-score in the amygdala of 6. (The amygdala is the only region to be remotely associated with the term.) "Arousal" is a bit better, with a reverse Z-score of 8 . The terms most likely to be associated with the amygdala are "emotional" ( $\mathrm{Z}=19)$, "neutral" ( $\mathrm{Z}=16)$, and "faces" $(\mathrm{Z}=15)$. The latter two represent control conditions and the most common visual cue to study emotion. Thus, as we discussed, the most conservative interpretation of amygdala activation in this experiment is as a biomarker of arousal. It should be noted that although peripheral measures of arousal, notably skin conductance response (SCR) are commonly used in humans (Harmon-Jones \& Harmon-Jones), these represent downstream physiological effects from events presumably originating in the brain. Apart from the difficulty of accurately measuring SCR in dogs - who only have sweat glands on their paws - its relationship to other measures of arousal in dogs is not well-validated (Rialland et al., 2012). The interpretation of amygdala activity, then, must be viewed in the context of the task itself (Adolphs; Singer; Zentall). 
The key difference is between the subject dog watching a fake-dog receive a treat versus a treat being deposited in a bucket. The bucket controls for the disappointment of not receiving the treat. Any additional activity must be due to the interaction with the fake dog. Several commentators have offered potential explanations other than proto-jealousy, echoing many of the interpretations we also noted. Many of these interpretations depend on formal definitions, which, in our view, is not an interesting debate. Taken at face value, the experiment demonstrated a covert arousal response to a doglike creature being fed. For some dogs at least, it is the "dogness" that drives this reaction. The fact that the statue is present constantly means that it must be the interaction itself that triggers the reaction. Is it jealousy in all the myriad human interpretations of the word? Probably not. But it is well within the possibility for resource guarding (for food and attention) - and that, we argue could form the basis of jealousy.

Is it the fakeness of the dog that is driving the amygdala response? (Horowitz et al.; Jiang et al.; Prato Previde \& Valsecchi; Silver \& Santos; Vonk). This is certainly a possibility, but it raises the question of why more aggressive dogs should be more aroused by the fakeness (see below). Some have questioned the validity of using fake dogs because one study could not replicate the original result (Harris \& Pouvost, 2014; Prato Previde et al., 2018). However, all these studies have shown an increase in dogs' attention to fake dogs when their owners interact with them. It is in the interpretation of what constitutes "jealousy behavior" that authors seem to disagree - which should make clear the limitations of behavior for reverse inference of emotional states: Behavior itself has fewer degrees of freedom than the brain does. In mathematical terms, behavior is "degenerate."

It is ironic, then, that while some commentators have criticized the use of a fake dog, the field of canine cognitive neuroscience has readily accepted the use of pictures on computer screens and auditory recordings as acceptable facsimiles of the real things. And yet, there is no evidence that dogs treat such abstracted representations in the same way humans do. A realistic statue of a dog is undoubtedly more likely to be recognized than a picture of a dog. Our results add further explanatory power via the correlation with other temperamental traits, as well as the potentially covert nature of arousal itself, as indexed by the amygdala.

\section{Individuality}

As in human fMRI studies, there is substantial heterogeneity in the neural responses. There are two distinct factors behind such variability: (1) anatomical variation and (2) functional variation. Anatomical variation arises when a brain structure differs in size or location across individuals; this can become problematic when averaging brains. The usual methods of aligning, transforming, and warping brains to fit a standardized template do not result in perfect alignment. One standard remedy is to "smooth" the resultant maps, increasing the likelihood that corresponding regions will overlap in a group analysis. Even in humans, this is an imperfect solution. The heterogeneity in size and morphology in dogs is much greater. However, subcortical structures - like the amygdala - tend to be less variable than the cortex and have the benefit of having discrete boundaries.

Although anatomical heterogeneity contributes to the variation in observed fMRI responses, the more interesting differences arise from functional causes. Different dogs may respond differently to the same stimulus, as is certainly the case here. Although several 
commentators offered alternative explanations for amygdala activity, only one highlighted the variability of their responses as a potential guide for therapeutic interventions (Howell). We agree. There is an unfortunate tendency in the canine literature to draw sweeping conclusions about dog cognition as if all dogs exhibit a given finding. There is clearly great variability in how they respond to experimental situations. That is what we observed with the antecedents of jealousy.

Our main finding was that dogs scoring higher in aggression on the C-BARQ had higher differential responses when the owner fed the fake dog. Although the sample size was small, assumptions of homoscedasticity were not violated (Vonk). Linear regression of the square of the residuals against DogAggr was not significant $(P=0.30)$. Similarly, the White test for heteroscedasticity was also nonsignificant $(P=0.60)$. Some have also criticized the use of the C-BARQ, or specific subscales (Overall; Serpell). It should be noted that although the C-BARQ is indeed a retrospective evaluation of the dog, it was done when the dogs entered the overall project, not for this particular experiment. Moreover, the experimenters have had years of experience with these dogs through their long-term participation in fMRI experiments. We have found that the C-BARQ subscales accurately capture our impression of the individual dogs. Those that score highly on Dog Aggression are indeed the dogs we have seen act aggressively in practice.

As noted above, amygdala activity is probably best thought of as a biomarker for arousal. Some dogs are more prone to arousal than others. The finding that this correlates with temperamental measures of dog-aggression suggests that there can be what we would call a "hair-trigger" amygdala. Even though the experiment was designed to set up a socially salient situation of potential resource loss, we think it is likely that it taps into a common pathway through the amygdala for a variety of high-arousal behaviors. Aggressive behavior - although often used for social signaling itself - generally requires arousal to be credible. The behavioral manifestations of aggression are well-described, but the neural substrates are not. Our results show that it is possible to have neural activation of arousal circuits even in the absence of overt aggression, but individuality is key. Not all dogs reacted this way. In the absence of amygdala activation, can we conclude that a dog does not care about threats to resources? It is difficult to answer this question because one dog might react with high arousal while another may enter a depressive state, perhaps with lowered amygdala activity. However, we are not aware of a metric that captures dogs with "mopey" temperaments.

\section{Conclusion}

It is gratifying to see that science has moved past the question of whether dogs have emotions and entered an era of investigation into the nature of those emotions. It is inevitable that the same problems that have plagued the study of human emotions problems of definition and measurement - will apply to dogs. And while humans can describe their emotions with words, dogs cannot. Here, and in the target article, we have argued for the role of neuroimaging in the measurement of covert arousal as a way to gain insight into one component of these hidden states. We suggest this approach offers significant advantages over those that rely exclusively on behavior. 
Funding: This work was supported by the Office of Naval Research (N00014-16-1-2276). ONR provided support in the form of salaries for authors, scan time, and volunteer payment, but did not have any additional role in the study design, data collection and analysis, decision to publish, or preparation of the manuscript.

Competing Interests: G.B. owns equity in Dog Star Technologies and developed technology used in some of the research described in this paper. The terms of this arrangement have been reviewed and approved by Emory University in accordance with its conflict of interest policies.

\section{References}

Abdai, J., \& Miklósi, Á. (2018) Displaying jealous behavior versus experiencing jealousy. Animal Sentience 22(21).

Adolphs, R. (2018) What would we like to know by imaging the brains of dogs? Animal Sentience 22(14).

Apter, A., Van Praag, H. M., Plutchik, R., Sevy, S., Korn, M., \& Brown, S. L. (1990) Interrelationships among anxiety, aggression, impulsivity, and mood: A serotonergically linked cluster? Psychiatry Research, 32(2), 191-199.

Cook, P. F., Spivak, M., \& Berns, G. (2016) Neurobehavioral evidence for individual differences in canine cognitive control: An awake fMRI study. Animal Cognition, 19(5), 867-878.

Cook, P., Prichard, A., Spivak, M., \& Berns, G. S. (2018) Jealousy in dogs? Evidence from brain imaging. Animal Sentience 22(1).

Culotta, C. M., \& Goldstein, S. E. (2008) Adolescents' aggressive and prosocial behavior: Associations with jealousy and social anxiety. Journal of Genetic Psychology, 169(1), 2133.

Ekman, P. (1992) An argument for basic emotions. Cognition \& Emotion, 6(3-4), 169-200.

Grotpeter, J. K., \& Crick, N. R. (1996) Relational aggression, overt aggression, and friendship. Child Development, 67(5), 2328-2338.

Harmon-Jones, E., \& Harmon-Jones, S. K. (2018) On jealousy, envy, sex differences and temperament in humans and dogs. Animal Sentience 22(8).

Harris, C. R., \& Prouvost, C. (2014) Jealousy in dogs. PloS One, 9(7), e94597.

Horowitz, A., Franks, B., \& Sebo, J. (2018) Fill-in-the-blank-emotion in dogs? Evidence from brain imaging. Animal Sentience 22(20).

Howell, T. J. (2018) Can neuroimaging in dogs have practical implications? Animal Sentience 22(6).

Jiang, Y., Huttunen, A. W., \& Platt, M. L. (2018) Can a dog be jealous? Animal Sentience 22(11).

LeDoux, J. E. (2014) Coming to terms with fear. Proceedings of the National Academy of Sciences, USA, 111(8), 2871-2878.

Lerner, J. S., Li, Y., Valdesolo, P., \& Kassam, K. S. (2015) Emotion and decision making. Annual Review of Psychology, 66.

Nagel, T. (1974) What is it like to be a bat? Philosophical Review, 83(4), 435-450.

Overall, K. L. (2018) Dogs aren't jealous - they are just asking for accurate information. Animal Sentience 22(17).

Panksepp, J. (2004) Affective neuroscience: The foundations of human and animal emotions. Oxford University Press. 
Parkinson, B. (1996) Emotions are social. British Journal of Psychology, 87(4), 663-683.

Poldrack, R. A. (2006) Can cognitive processes be inferred from neuroimaging data? Trends in Cognitive Sciences, 10(2), 59-63.

Poldrack, R. A. (2011) Inferring mental states from neuroimaging data: From reverse inference to large-scale decoding. Neuron, 72(5), 692-697.

Prato Previde, E., \& Valsecchi, P. (2018) What is it like to be a jealous dog? Animal Sentience 22(16).

Prato Previde, E., Nicotra, V., Pelosi, A., \& Valsecchi, P. (2018) Pet dogs' behavior when the owner and an unfamiliar person attend to a faux rival. PloS One, 13(4), e0194577.

Rialland, P., Authier, S., Guillot, M., del Castillo, J. R. E., Veilleux-Lemieux, D., Frank, D., Gauvin, D., \& Troncy, E. (2012) Validation of orthopedic postoperative pain assessment methods for dogs: A prospective, blinded, randomized, placebo-controlled study. PLoS One, 7(11), e49480.

Serpell, J. A. (2018) Jealousy? or just hostility toward other dogs? The risks of jumping to conclusions. Animal Sentience 22(13).

Sharpsteen, D. J., \& Kirkpatrick, L. A. (1997) Romantic jealousy and adult romantic attachment. Journal of Personality and Social Psychology, 72(3), 627.

Silver, Z. A., \& Santos, L. R. (2018) Understanding dogs' neural responses in a food-giving paradigm. Animal Sentience 22(15).

Singer, P. (2018) Finding the green-eyed monster in the brain of a dog. Animal Sentience 22(3).

Smith, R. H., \& Kim, S. H. (2007) Comprehending envy. Psychological Bulletin, 133(1), 46.

van Kleef, G. A. (2009). How emotions regulate social life: The emotions as social information (EASI) model. Current Directions in Psychological Science, 18(3), 184-188.

van Kleef, G. A. (2018) Emotional reactions in non-human animals and social-functional theories of emotion. Animal Sentience 22(12).

Vonk, J. (2018) Researchers, not dogs, lack control in an experiment on jealousy. Animal Sentience 22(2).

Webb, C. E., \& de Waal, F. B. M. (2018) Situating the study of jealousy in the context of social relationships. Animal Sentience 22(22).

White, G. L., \& Mullen, P. E. (1989) Jealousy: Theory, research, and clinical strategies. Guilford Press.

Zentall, T. R. (2018) Jealousy, competition, or a contextual cue for reward? Animal Sentience 22(4). 